\title{
Agent-Based Co-Modeling of Information Society and Wealth Distribution
}

\author{
Fayçal Yahyaoui*, Mohamed Tkiouat \\ IFE-Lab, Laboratory of Research in Applied Mathematics, Ecole Mohammadia d'Ingenieurs, \\ Mohammed V University, Rabat, Morocco
}

\begin{abstract}
With empirical studies suggesting that information technology influence wealth distribution in different ways, and with economic interactions and information technology adoption being two complex phenomena, there is a need for simulation approach that addresses the whole complexity of the issue without being too costly in terms of computations and without ignoring relevant empirical facts in defining the behavior of different agents.. While this problem seems to require a bottomup approach using agent-based modeling, further complexity levels in managing the heterogeneous agents in space and time and an appropriate separation in domain areas show its limitations in practice. In this paper we illustrate the use of novel multi-level agent based concepts on this socio-economic issue, by considering our studied phenomenon as an interference of multiple simple other phenomena, namely a basic producer/consumer economy and a diffusion of information model. Such an approach involves writing models following a formalism allowing compatibility and exchange of variables, in addition to implementing appropriate synchronization algorithms. Our simulation used Levelspace, a recent extension project of Netlogo simulation tool combined with data exploration tools but the patterns described are generic and can be implemented in other simulation tools. Indeed, our case study offers a building block for a framework that can investigate wealth dynamics and other analogue cases with influence between models. Our approach successfully validates against empirical macro-trends in the distribution of wealth and other social patterns. Thanks to its flexibility in conducting experiments, we could reduce the hypotheses that restricted previous models from conducting a multi-dimensional analysis for the Gini index and enabled solving conflicting research issues.
\end{abstract}

Keywords-Agent-based modeling; computational economics, multi-level; complex systems; parallel computing

\section{INTRODUCTION}

Economics is considered a complex system where interactions and behavior of micro-elements produce macro phenomena that cannot be understood by a top-down linear decomposition[1]. Agent based modeling is an alternative to statistical modeling by trying to understand this micro-macro link with bottom-up experiments where simple actions of agents are defined with minimal assumptions based on empirical facts to study resulting phenomena. In its basic form agent-based simulation is still limited to address the complexity of economics and other complex phenomena that have different levels of representations. [7]

In fact, a level can be defined as a point of view in a system, integrated in a model as a specific abstraction [8]. We can note that agents can act on different levels, for instance policy makers generally interact with conceptual aggregations of agents (companies, sectors) rather than atomic agents. Some elements of agent-based simulations can also be influenced or constituted by interactions related to another phenomena represented by an agent-based approach. [8]

Many agent-based modelling tools exist to describe models and conduct such experiments [2], they differ mainly in user experience and software environment. Concurrently, research is continuously conducted on different aspects either in simulation theory to provide formalisms that can generalize and fit different modeling situations and also on improving the performance, flexibility, of the tools to be able to reproduce real phenomena

For wealth distribution, we can observe that a small change in a population can induce unpredictable change in wealth curves in the populations with simplest economic interactions [4], which explains the relevance of agent-based modelling for this issue as mathematical equations become quickly unsolvable if we consider the constraints and irregularities: an indicator that such a system falls into the complex category.

The aim of this paper is to show how recent multi-level agent based concept can offer effective ways and new perspective to study classical complex economic issues of interest rather than making the simulation more complicated or adding hypotheses that restrict the conduction of artificial experiments. We illustrate our paradigm through a multi-level simulation that assesses the impact of information society on wealth distribution where the design and performance outperform previous works.

The paper is organized as follows: In section 2, we review briefly the related literature, in section 3 we describe the methodology adopted and show why it's relevant for the phenomena addressed in this paper. In section 4 , we describe the model and its specifications to finally discuss the results obtained and the approach in section 5

\section{RELATED LITERATURE}

Bottom-up approaches using agent based modeling and experiments on artificial societies to understand economic have offered an important alternative to classical economics in what is known as agent based computational economics [1] where many simulations manage to better understand phenomena traditionally explained by top down approach such as macroeconomic policies [11] . 
The limitations of traditional models lie mainly in considering the agents as heterogeneous and perfectly rational while the empirical facts say otherwise. Those models also ignore the fact that a system can present emergent phenomena unexplained by summing its different parts.

Wealth distribution, which also appears in many agent based simulations is a particularly complex issue due to its interdisciplinary nature [3] where modeling research aims to understand how different social and economic factors influence wealth inequalities. For instance, the impact of technology on wealth distribution from an industrial perspective where technological advances affect productivity and employment was investigated in one of these models [2]. While the model showed how a behavioral paradigm outperformed traditional models by being able to define heterogeneous agents with a bounded rationality and reproducing emergent historical changes, the structure of the model remained specific to the studied case and does not offer any design pattern that can be generalized to similar situations.

Also, with the large diffusion of information technology, many agent-based models try to simulate information society trends and other connected phenomena such as smart grids [15]. However, most research on the influence of modern technologies on wealth distribution remains based on descriptive statistics [10], mainly due the multi-level nature of the problem where the first one belongs to microeconomics and the second one belongs to complex network theory. In fact, recent literature acknowledges that multi-level agent based modeling is relevant to socioeconomic systems with different points of view which is exactly the case for our problematic. For this purpose, many generic or domainspecific frameworks using different approaches try to address the multi-level issue by considering the levels as either aggregations of agents or by connecting models or by defining a global system reaction to each agent action [Siebert, 2010]. Those concepts have been able to conduct successful simulations of multi-level complex phenomena such urban growth, cancer modeling, and many other complex phenomena [7].

However, there are no examples in the literature of applications of these recent concepts to simulations of socioeconomic phenomena. Limited examples of multi-level agent-based simulations can be found but they have in common that all the levels are included as agent interaction with other agents without a separation of scale or complexity thus increasing the computational cost of the simulation

\section{MethodOLOGY}

\section{A. Formalism}

Let $\mathbf{M}$ be a model defined by a group of agents interacting using specific procedures in an environment $\mathbf{E}$. Let 's assume that we manage to isolate in the execution of the model some procedures involving only groups of agents without the others. The execution of the model would be equivalent to (M1, R1, M2, R2,...Mi, Ri) where Mi are the isolated sub models and $\mathbf{C i}$ are model subroutines that could not be reified as a sub model. Depending on the complexity of these subroutines, they could be or not integrated as dynamic variables of the environment or approximated using statistical laws. Let's take for example the case of an agent that increases the price of a good periodically where the good is not an agent property, this can be easily translated into a variable of the environment following an arithmetic sequence. In other cases, we can also replace a decision mechanism in these subroutines with decisions based on observations of the environment, as in an inventory of sales of some category of products the results can suggest that some agents are doing financially well. While it is not always feasible to be able to view a phenomenon as an interference of other phenomenon's in case those subroutines have considerable complexity, our goal is to reach a situation describe by Fig. 1 .

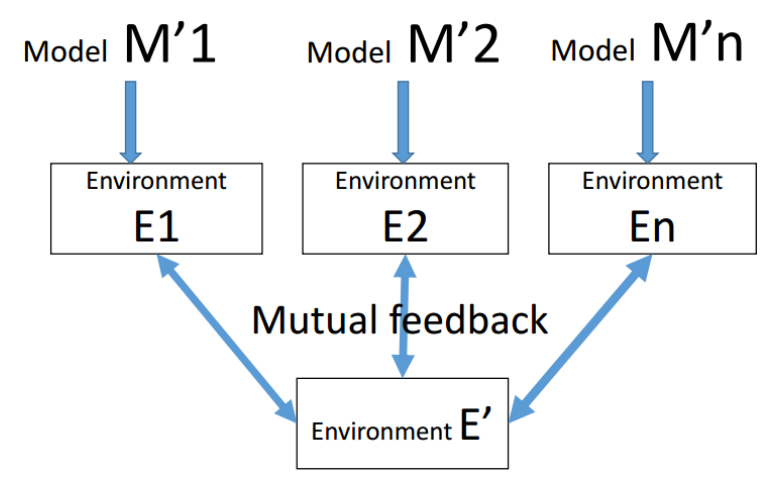

Fig. 1. Model Decomposition.

M'i describes domain specific versions of the sub models Mi with respective environments Ei that can interact with the dynamic environment. An analogy of this pattern exists in signal processing where a noisy signal can be decomposed to known simpler signals. In our case the signal is the wealth distribution. This distribution results from interactions of different agents in the economy (consumers, producers,) as specified by other models. We can note however that this socioeconomic factor can be influenced by many different dynamics of society whether behavioral or economic which can be themselves complex and can't be simple components of a model but require themselves to be modeled. The model mentioned in section 2 was constrained to make a simplistic formulation of technological progress to be able introduce it in the economic model.

For our study, modeling trends in a more connected society and wealth distribution dynamics are separate areas of expertise and we suppose a preexistence of model examples. We consider the connected society as a separate level that changes the environment of economic interactions where agents interact, which is reflected in a new kind of transactions, associations, goods and other economic possibilities due to distant communication and large availability of information

Linking such models however requires both conceptual and technical tools for information exchange and synchronization.

\section{B. Tools}

For our simulation we use Netlogo, one of the most used agent based platforms in different fields [6] 
Experiments in Netlogo simply define agent properties and procedures then run simulations for different inputs while monitoring outputs of interests, which can result in dataset for later analysis or simply acknowledge the existence of an emergent phenomenon.

The growing research trends to take multi-level aspects in agent based simulations had led the founders of Netlogo project to conduct a research project that led to the development of Levelspace [9], an extension that allows users to take into consideration the multi-level aspects in different ways. This extension can call other simulations launched simultaneously to either connect different phenomena or reify agents in the simulation as resulting from another simulation.

A classic example is the wolf sheep predation model, where Levelspace can make the grass growth controlled by a climate change model that would have made the simulation unmanageable if included in the same model. Levelspace with other frameworks views the multi-level aspects in a model as a society of interacting models [9].

For our example, we consider informations society changes happening in a different level and we connect it to a classical economic interactions model. The "introduceICT" button in the first part of Fig. 2 below launches the model in the second part of the figure where we can see green nodes representing the environment getting connected by red segments to diffuse information.

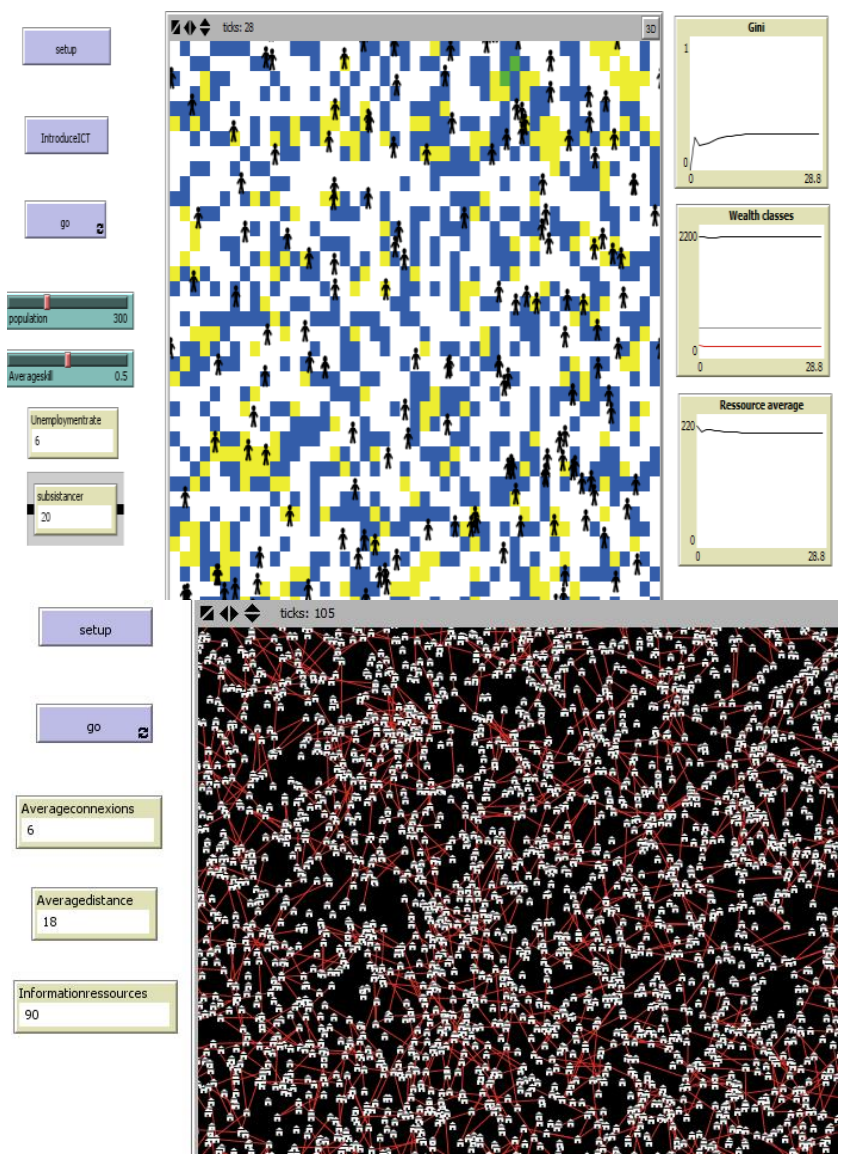

Fig. 2. Information Society Model Connected with Economy Model.

\section{THE MODEL}

\section{A. Agents and Actions}

This model is based on classical agent-based models of wealth distribution with individuals having basic economic interactions with each other and with the environment.

We consider however that the skill of an agent can be improved by a resource of type information. We list the properties of the agents below.

Resource agent: (represented by the patch)

- vision : represents how the radius of accessible resources

- sellable: Boolean variable that represents whether the resource can be sold or not

- resource type: either material or information or subsistence

- value: a quantification of the value of the resource

Individual agent:

- wealth: variable that represents the global wealth of the individual

- skill: skill of the individual which determines the ability to extract a resource

- material: quantity of material resources

- subsistence: quantity of subsistence resources

In this model according to the flow chart in Fig. 3 agents harvest the maximum resources that their location allows them to, giving priority to subsistence resources. An excess of resources allows the individuals to do other operations such as employing starving agents to harvest for them or selling and transforming resources. We can note that the execution of this model after significant steps shows an increase in Gini index and a relative stabilization in the long run which is consistent with other related models [2]

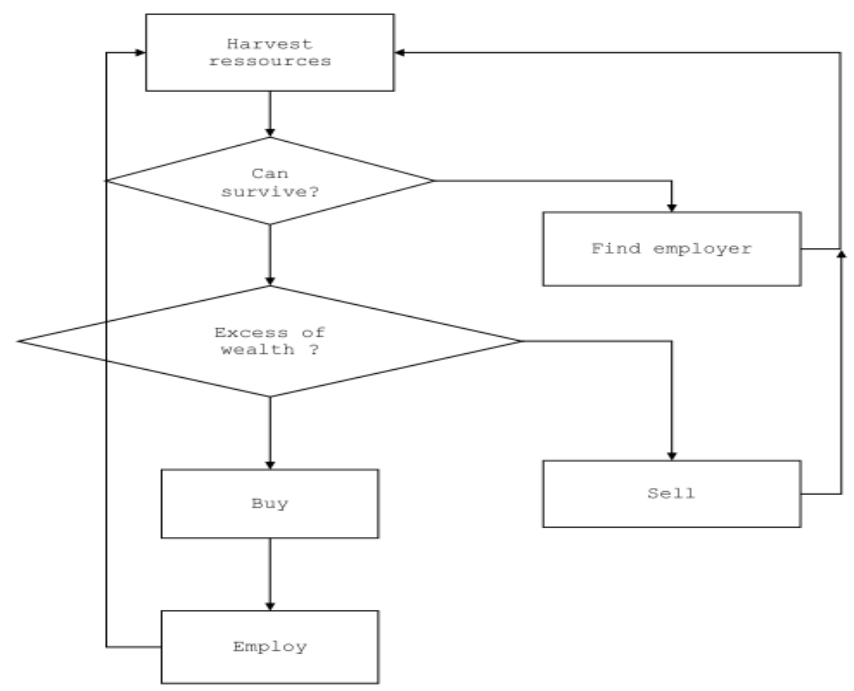

Fig. 3. Flow Chart of the Economy Model. 
Parallel to this model is the information society model based of diffusion in a network [5]. In this case, it simulates how information can be more accessible when resource nodes connect to each other. The model has one node agent. In each step arbitrary nodes connect with each other and affect the vision of other nodes proportionally to numbers of continuously connected nodes and proximity of material resources.

Below is an algorithm of the procedure "updateinfo" that updates the properties of node in each execution step. Nodes with more than 5 neighbors pointing to material resources become sellable and the vision is incremented while traversing the connected neighbors that are connected to more than 10 nodes. We refer in the following algorithm to the resource who are considered connected to a parent resource as "linkneighbors" and to select a resource based on the maximality of a criteria by max-one-of.

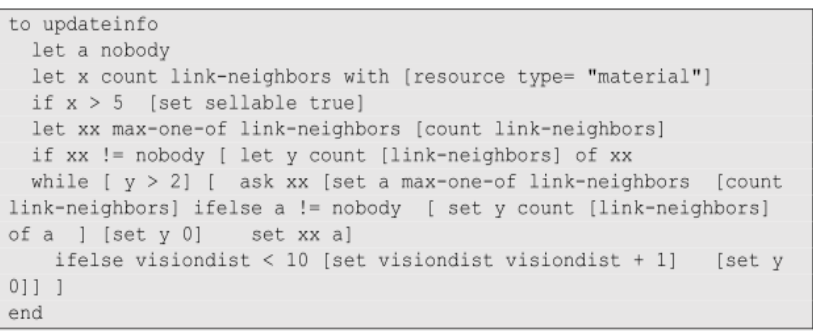

This algorithm is not based on a graph structure but its complexity is not exponential due to the Netlogo graph methods implemented in the link agent.

\section{B. Computational Considerations}

To assess the impact of information technology by connecting the environment of the second model on wealth distribution of the first model, we assimilate that the connections will link resources of the environment, and the access to resources will become transitive in a defined radius .A diffusion of information will also impact the skills of the individuals. In each step, the properties of resources are sent to the second model as properties of the nodes, a step of connection is executed then the new values of the properties affected by this connection are sent back to the economy model as shown in Fig. 4, and so on.

One step of a model can refer to multiple runs depending on the time scale proportion of the two models. For instance, the exchange and spread of information occurs with more frequency than the economic transactions, and we set the number of runs of the latter to 3. Assuming the existence of appropriate datasets, a proportion factor of communication time and volume of transactions normalized by a time /information exchange indices would be a more accurate estimation of this number of runs.

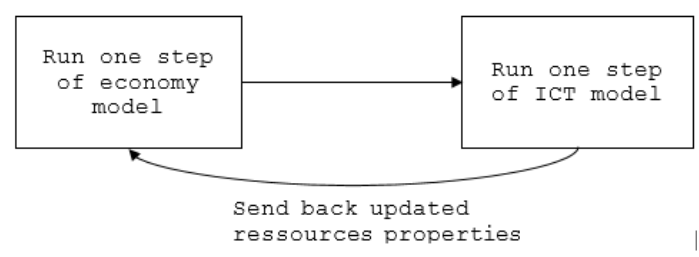

Fig. 4. Synchronization between Models.
Below is the synchronization algorithm that transfers the properties of resources to nodes in the second model to be influenced by connection.

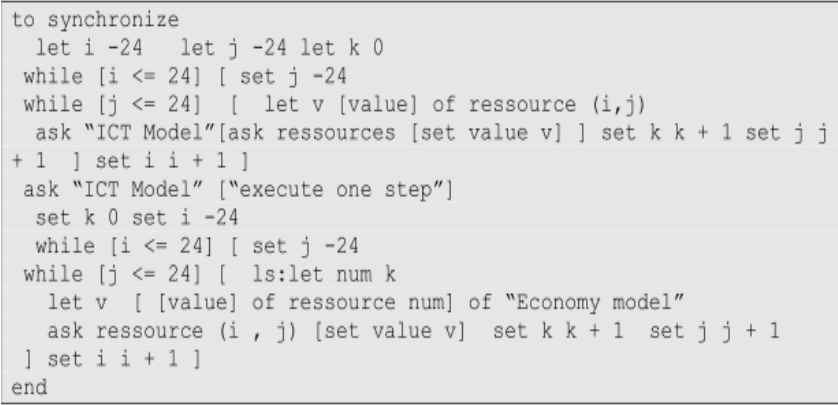

This algorithms loop on indices of the resources to transfer them to the other to address a limitation of Netlogo in concurrent programming, which does impact this specific model.

However in other cases, a sequential synchronization can make the model dysfunctional since the agents react to a state in the environment that was modified by another agent while they were supposed to react simultaneously to the unmodified state. The advantage of our design that is making the synchronization process part of the simulation rather than external is compatible with the transactional memory mechanism that places locks on variables mutually influenced by other agents as specified in Hlogo [12].

Nonetheless, computational efficiency issues might arise when the number of agents of transactions is high. The solution is to define "meso" entities in the model as groups of agents having the same behavior as in pedestrian simulation models, such a solution is also totally compatible with our design since the inter-model communication happens only in the environment and is not affected by a change of agents.

\section{NUMERICAL ANALYSIS}

\section{A. Validation and Calibration}

The values of the inputs of the model to conduct experiments were chosen according to different patterns suggested by different studies to ensure that the analysis is independent from input functions.

The experiments were conducted according to Table. 1 scenario:

TABLE I. EXECUTION SCENARIO

\begin{tabular}{|l|l|l|l|l|}
\hline & $\begin{array}{l}\text { Minimum } \\
\text { value }\end{array}$ & Step & $\begin{array}{l}\text { Maximum } \\
\text { value }\end{array}$ & Law \\
\hline Population & 100 & 20 & 1000 & Linear \\
\hline wealth & 5 & None & 1000 & Normal \\
\hline $\begin{array}{l}\text { Resource by } \\
\text { individual ratio }\end{array}$ & 2 & 0.5 & 10 & Linear \\
\hline Propagation speed & 1 node/step & & 10 nodes & $\begin{array}{l}\text { Time } \\
\text { Linear }\end{array}$ \\
\hline $\begin{array}{l}\text { Unemployment } \\
\text { rate }\end{array}$ & 4 & & 25 & Random \\
\hline
\end{tabular}


Since the goal of the model is to search for macro patterns and not reproduce a real case study of a specific country or society, we opted for a level 2 validation [13] according to the constant following macro-properties in our executions:

-Existence of different wealth classes in a pyramidal structure

-Existence of economic cycles reflected in the wealth of the bottom classes

-Unemployment rate always above $4 \%$ to ensure

-Subsistence resources remaining the main form of resources by a $20 \%$ percentage.

\section{B. Analysis}

We conducted our analysis based on categories environment that launches the second model once its characteristics were detected by CART trees implemented in $\mathrm{R}$ statistical language which can communicate with Netlogo through the RNetlogo extension [14] . We considered the Gini index as the main measure of wealth distribution of a population. It is computed as the ratio of the cumulative wealth of each percentage of the population and the same wealth in a case of equal distribution.

We list below the categories of the our environments that were influenced by the introduction of information technology

- Non-egalitarian society: characterized by a transfer of wealth to the wealthier agents and an increase in the looking for survival pool.

We note in the plots of this case in Fig. 5 that the introduction of information technology to the environment inverses the tendency of the Gini curve for a short period then goes back to its initial shape. The same tendency can be observed in resource values where the average value increased temporarily then returned to its decreasing shape.

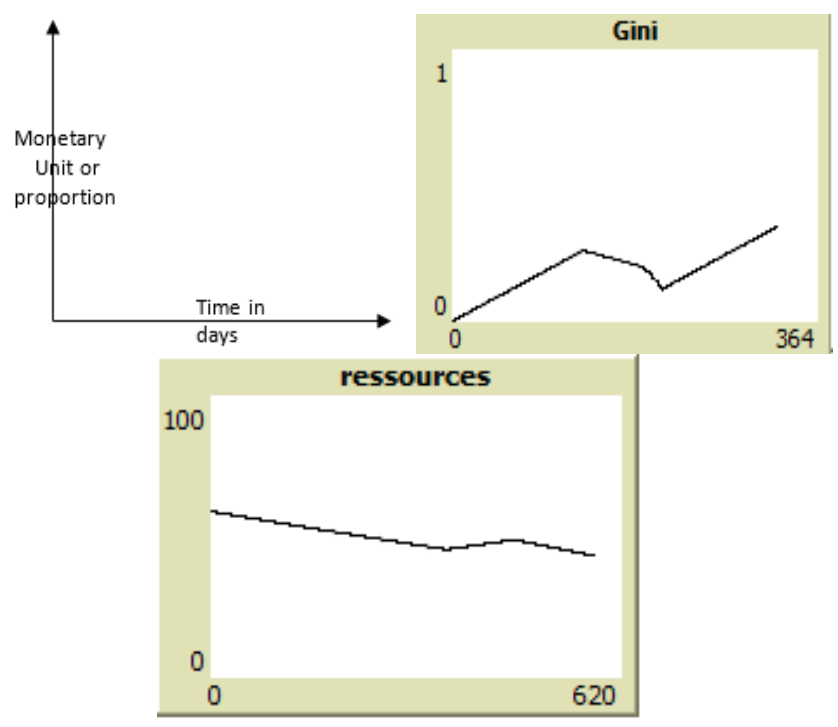

Fig. 5. Non-Egalitarian Society Plots.
- Egalitarian society: characterized by slight variations in cycles over an average of the Gini index."

In this society the introduction of information technology creates an immediate perturbation period shown in Fig. 6 then returns to its previous shape and the same thing can be said about resource values

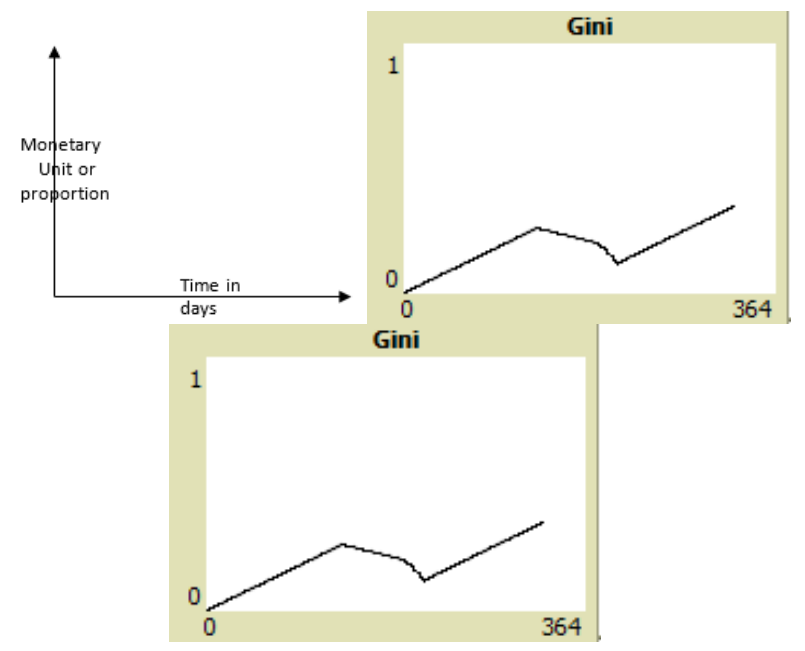

Fig. 6. Egalitarian Society Plots.

- Society with skilled individuals: characterized by a high skill average

The occurred mainly in the wealth pyramid where more than $50 \%$ of wealth became shared by more individuals. However this was not reflected in the Gini curve which suggests that the unskilled individuals got poorer.

- Society with abundance of material resources:

We noted in this category of society a tendency to more wealth repartition in the bottom of the pyramid but not in Gini curves which suggests that the richest individuals got even richer (Fig. 7).

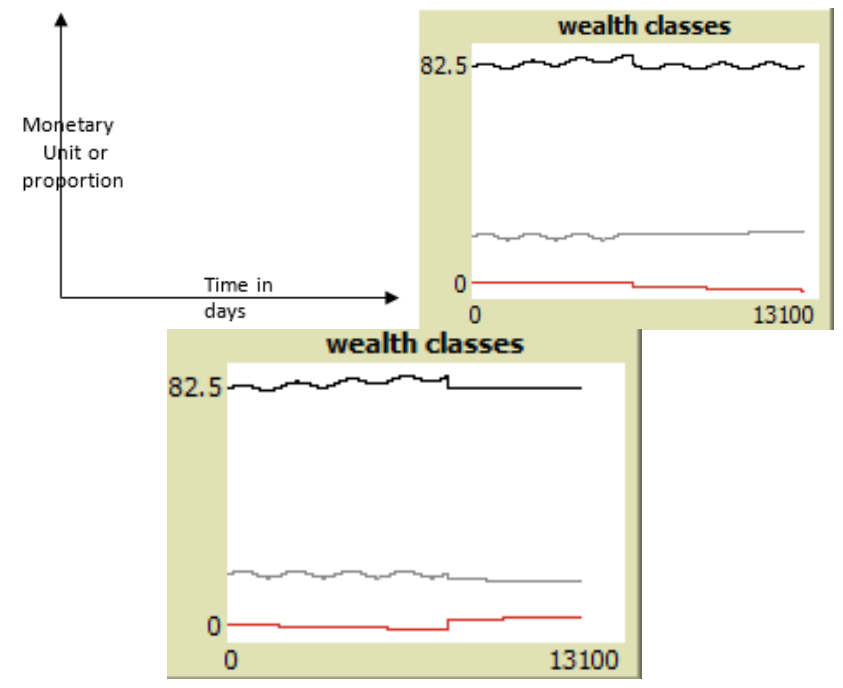

Fig. 7. Wealth Classes Plots. 


\section{CONCLUSION}

We can assert from the previous analysis that our simulation suggests that information technology has a negligible impact on wealth distribution. This is compatible with the fact that no conclusion can be drawn from empirical evidence where some case studies suggest an increase in inequalities while others suggest a decrease [4]. Undoubtedly, information society offers new opportunities for the less wealthy, but it also offer means to the wealthier for better exploitation of resources and a wider access to labor as suggested by reporters in our model. The stabilization of Gini curve is an emergent phenomena of our model and supports the theory that information technology in the long run contributes to the stabilization of wealth distribution which is still a positive effect. In comparison with models that have one level, our model being composed of two simple components allows change in any of the models without affecting many variables, it also encapsulates the non-relevant variables for our experiments and allows us to explore flexibly different possibilities of modern technology influence with a significant gain in computation costs.

\section{REFERENCES}

[1] Naciri, Nawfal \& Tkiouat, Mohamed. Economic agent based models: Review. International Journal of Applied Engineering Research. 11. 5492-5502. 2016

[2] Damaceanu, R.C.. An Agent-based Computational Study of Wealth Distribution in Function of Technological Progress Using Netlogo. American Journal of Economics . 2018

[3] Sanchari Goswami, Agent based models for wealth distribution with preference in interaction ,Parongama Sen Physica A: Statistical Mechanics and its Applications 415 - August 2014.

[4] Davies, Jim \& Fortin, Nicole \& Lemieux, Thomas. Wealth Inequality: Theory, Measurement, and Decomposition. Canadian Journal of Economics/Revue canadienne d'économique. 10.1111/caje.12313. 2017
[5] D. Gruhl, R. Guha, D. Liben-Nowell, and A. Tomkins, "Information diffusion through blogspace." In Proceedings of the 13th international conference on World Wide Web, pp. 491-501. ACM, 2004.

[6] Naciri, Nawfal \& Tkiouat, Mohamed. Agent-based platforms comparison. 10. 34224-34229. 2015

[7] Gil-Quijano, J., Louail, T., and Hutzler, G. From biological to urban cells: Lessons from three multilevel agent-based models. In Desai, N., Liu, A., and Winikoff, M., editors, Principles and Practice of MultiAgent Systems, volume 7057 of Lecture Notes in Computer Science, pages $620-635$. Springer. 2008

[8] Soyez, J.-B., Morvan, G., Merzouki, R., Dupont, D., and Kubiak, P. Multi-agent multi-level modeling - a methodology to simulate complex systems. In Proceedings of the 23rd European Modeling \& Simulation Symposium. 2011

[9] Hjorth, A., Head, B., \& Wilensky, U. LevelSpace NetLogo Extension. http://ccl.northwestern.edu/levelspace. Center for Connected Learning and Computer-Based Learning. Evanston, IL. Retrieved from www.github.com/NetLogo/LevelSpace .2015

[10] Sanghamitra Bandyopadhyay, Are Mass Media and ICTs associated with Inequality and Poverty? Queen Mary University of London, Phd Thesis 2015.

[11] Deissenberg, Christophe, Vanderhoog, Sander, \& Dawid, Herbert. EURACE: A massively parallel agent-based model of the European economy. Applied Mathematics and Computation, 204(2), 541-552. 2008

[12] Bezirgiannis, Nikolaos \& Prasetya, Wishnu \& Sakellariou, Ilias. HLogo: A Haskell STM- Based Parallel Variant of NetLogo. Advances in Intelligent Systems and Computing · July 201897- 119. 10.1007/978-3319-69832-8_7. 2018

[13] AXTELL, R.L. \& Epstein, J. M. . "Agent-Based Modeling: Understanding Our Creations". Bulletin of the Santa Fe Institute. page: 28-32, Winter 1994

[14] Thiele, J.R Marries NetLogo: Introduction to the RNetLogo Package. Journal of Statistical Software, 58(2), 1 - 41. doi:http://dx.doi.org/10.18637/jss.v058.i02 .2014

[15] Vaubourg, Julien \& Presse, Yannick \& Camus, Benjamin \& Bourjot, Christine \& Ciarletta, Laurent \& Chevrier, Vincent \& Tavella, jeanphilippe \& Morais, Hugo \& Deneuville, Boris \& Chilard, O.. Smart grids simulation with MECSYCO. Lecture Notes in Artificial Intelligence (Subseries of Lecture Notes in Computer Science). 9086. 10.1007/978-3-319-18944-4-37.2015 